\title{
EXTREMAL PROBLEMS FOR A CLASS OF FUNC- TIONALS DEFINED ON CONVEX SETS ${ }^{1}$
}

BY ZEEV NEHARI

Communicated by Felix Browder, February 20, 1967

1. Let $X=(X, \Sigma)$ be a measurable space, and let $\tau$ be a class of positive measures $\mu$ defined on $\Sigma$. We consider a set $H$ of nonnegative functions belonging to $L^{p}(\mu)$ on $X$ for all $\mu \in \tau(1 \leqq p<\infty)$, and we denote by $C(H)$ the convex hull of $H$. If $\sigma$ is an arbitrary positive measure on $X$, we define the functional $\Lambda(r)\left(r \in C(H), L^{1}(\sigma)\right)$ by

$$
\Lambda(r)=\sup _{\mu \in \tau}\left[\int_{X} r^{p} d \mu\right]^{1 / p} / \int_{X} r d \sigma .
$$

The following result is a useful tool in the treatment of numerous extremal problems involving eigenvalues of differential and integral equations.

Theorem I. If $\Lambda(r)$ is the functional defined by (1), then

$$
\sup _{r \in C(H)} \Lambda(r)=\sup _{s \in H} \Lambda(s) .
$$

The proof of (2) is very simple. Since $H \subseteq C(H)$, (2) will follow from the inequality

$$
\sup _{r \in C(H)} \Lambda(r) \leqq \sup _{s \in H} \Lambda(s),
$$

and it is sufficient to establish (3) for finite sums of the form

$$
r=\alpha_{1} s_{1}+\cdots+\alpha_{n} s_{n}, \quad \alpha_{k}>0, \quad \sum_{k=1}^{n} \alpha_{k}=1, \quad s_{k} \in H .
$$

By Minkowski's inequality, we have

$$
\left[\int_{X} r^{p} d \mu\right]^{1 / p} \leqq \sum_{k=1}^{n} \alpha_{k}\left[\int_{X} s_{k}^{p} d \mu\right]^{1 / p}
$$

and thus, by (1),

\footnotetext{
${ }^{1}$ Research sponsored by the Air Force Office of Scientific Research, Office of Aerospace Research, United States Air Force, under Grant No. 28.
} 


$$
\left[\int_{X} r^{p} d \mu\right]^{1 / p} \leqq \sum_{k=1}^{n} \alpha_{k} \Lambda\left(s_{k}\right) \int_{X} s_{k} d \sigma .
$$

Since this holds for all $\mu \in \tau$, it follows from (1) and (5) that

$$
\begin{aligned}
\Lambda(r) \int_{X} r d \sigma & \leqq \sum_{k=1}^{n} \alpha_{k} \Lambda\left(s_{k}\right) \int_{X} s_{k} d \sigma \\
& \leqq \sup _{s \in H} \Lambda(s) \sum_{k=1}^{n} \alpha_{k} \int_{X} s_{k} d \sigma \\
& =\sup _{s \in H} \Lambda(s) \int_{X} r d \sigma .
\end{aligned}
$$

Thus,

$$
\Lambda(r) \leqq \sup _{s \in H} \Lambda(s)
$$

if $r$ is of the form (4). Since these functions are dense in $C(H)$, this implies (3) and completes the proof of Theorem I.

2. As an example of a functional which can be brought into the form (1), we consider the lowest eigenvalue $\lambda=\lambda(R)$ of the differential system

$$
y^{(2 n)}-(-1)^{n} \lambda R(x) y=0, \quad U(y)=0, \quad(\lambda=\lambda(R))
$$

where $R>0, R \in L^{1}$ on an interval $[a, b], U(y)=0$ is a set of selfadjoint boundary conditions, and $n$ is a positive integer. By classical results, $1 / \lambda(R)=\sup \int_{a}^{b} R d \mu$, where $d \mu=u^{2}(x) d x$ and $u(x)$ ranges over the class of functions with the following properties: (a) $u$ satisfies the conditions $U(u)=0$; (b) $u^{(n)}$ is of class $L^{2}$ on $[a, b]$ and is normalized by the condition $\int_{a}^{b}\left[u^{(n)}\right]^{2} d x=1$. In this case, we thus have

$$
\left[\Lambda\left(R^{1 / p}\right)\right]^{-1}=\lambda^{1 / p}(R) \int_{a}^{b} R^{1 / p} d \sigma
$$

and Theorem I shows that

$$
\inf _{R^{1 / p} \in C(H)} \lambda^{1 / p}(R) \int_{a}^{b} R^{1 / p} d \sigma=\inf _{T^{1 / p} \in H} \lambda^{1 / p}(T) \int_{a}^{b} T^{1 / p} d \sigma .
$$

If the value of the right-hand side of (8) can be found, (8) thus provides the exact lower bound for the expression (7), where $R$ ranges over $C(H)$ or over a subset of $C(H)$ which contains $H$. 
3. The use of Theorem I as a source of estimates for functionals $\Lambda(r)$ is most likely to be successful in the case of convex sets $C(H)$ which are spanned by sets $H$ of functions of very simple type. There are many such sets which are of interest in the applications. Two well-known examples are:

(a) the class of bounded nonincreasing nonnegative functions on an interval $[a, b]$; in this case $H$ may be identified with the set of functions $A \chi_{t}(t \in(a, b])$, where $A$ is a suitable positive constant and $\chi_{t}$ is the characteristic function of the interval $[a, t]$;

(b) the class of nonnegative concave functions on an interval $[a, b]$; this class is spanned by the functions $g(x, t)(t \in[a, b])$, where $g(x, t)=A(x-a)(b-t)$ for $x \in[a, t]$ and $g(x, t)=A(t-a)(b-x)$ for $x \in[t, b]$.

Another example of this type-which does not seem to be found in the literature-is described in the following statement.

Theorem II. Let $(X, \Sigma, \mu)$ be a finite positive measure space, and let $K=K(m, M, \eta)$ be the class of measurable functions $F$ on $X$ for which

$$
-\infty<m \leqq F \leqq M<\infty
$$

and

$$
\int_{X} F d \mu=[\eta M+(1-\eta) m] \mu(X)
$$

$(0 \leqq \eta \leqq 1)$, where $m$ and $M$ are, respectively, the essential infimum and the essential supremum of $F$ on $X$.

If $H$ denotes the subset of $K$ consisting of the functions $g=m$ $+(M-m) \chi\left(X_{0}\right)$, where $X_{0} \subset X, \mu\left(X_{0}\right)=\eta \mu(X)$, and $\chi\left(X_{0}\right)$ is the characteristic function of $X_{0}$, then $K \subset C(H)$.

If we set $F=m+(M-m) f,(9)$ and (10) take the form $0 \leqq f \leqq 1$ and

$$
\int_{X} f d \mu=\eta \mu(X)
$$

respectively. It is thus sufficient to prove Theorem II for the case $m=0, M=1$. Another simplification which can be made is the assumption that $f$ be a step-function which takes only the values $0, \epsilon, 2 \epsilon, \cdots, N \epsilon$, where $\epsilon N=1$ and $N$ is an arbitrary positive integer. Indeed, $f$ may be approximated by functions $f^{*}$ defined by setting $f^{*}=\epsilon k$ on the subset of $X$ on which $\epsilon(k-\theta)<f \leqq \epsilon(k+1-\theta)$, where $\theta$ is a number in $(0,1)$, and $k=0,1, \cdots, N$. Evidently, inf $f^{*}=0$, $\sup f^{*}=1$, and 


$$
-\epsilon \theta \mu(X)<\int_{X} f d \mu-\int_{X} f^{*} d \mu \leqq \epsilon(1-\theta) .
$$

Since $\int_{X} f^{*} d \mu$ is a continuous function of $\theta$, this shows that $\theta$ may be so chosen that $\int_{X} f^{*} d \mu=\int_{X} f d \mu$ and thus, by $\left(10^{\prime}\right), \int_{X} f^{*} d \mu=\eta \mu(X)$.

If $S_{k}$ denotes the subset of $X$ on which $f \geqq \epsilon k(k=1,2, \cdots, N-1)$, we have

$$
S_{k+1} \subseteq S_{k}, \quad k=1, \cdots, N-2
$$

and

$$
\epsilon N \mu\left(S_{N-1}\right) \leqq \int_{X} f d \mu \leqq \epsilon N \mu\left(S_{1}\right)
$$

Since, by $\left(10^{\prime}\right)$,

$$
\int_{X} f d \mu=\eta \epsilon N \mu(X)=\eta \mu(X) \sup f
$$

(12) implies that

$$
\mu\left(S_{N-1}\right) \leqq \eta \mu(X) \leqq \mu\left(S_{1}\right)
$$

We denote by $S_{1}^{*}$ a subset of $S_{1}$ for which

$$
\mu\left(S_{1}^{*}\right)=\eta \mu(X)
$$

and which, in addition, is such that

$$
S_{1}^{*} \supseteq S_{N-1}
$$

The right-hand inequality (14) shows that there are subsets $S_{1}^{*}$ of $S_{1}$ for which (15) holds and it follows from (11) and the left-hand inequality (14) that $S_{1}^{*}$ may be so chosen as to satisfy (16).

We now consider the function

$$
f_{1}=f-\epsilon \chi\left(S_{1}^{*}\right) \text {. }
$$

Since $S_{1}^{*} \subseteq S_{1}$, we have $f_{1} \geqq 0$. Because of (16), we have

$$
\sup f_{1}=\sup f-\epsilon=(N-1) \epsilon
$$

and, by (13) and (15),

$$
\int_{X} f_{1} d \mu=\eta \epsilon(N-1) \mu(X)=\eta \mu(X) \sup f_{1} .
$$


A comparison of (13) and (18) shows that the procedure leading from (13) to (18) can be repeated. There will thus exist a subset $S_{2}^{*}$ of $X$ such that the function

$$
f_{2}=f_{1}-\epsilon \chi\left(S_{2}^{*}\right)
$$

is nonnegative and satisfies

$$
\int_{X} f_{2} d \mu=\eta \epsilon(N-2) \mu(X)=\eta \mu(X) \sup f_{2} .
$$

By applying this process $N$ times, we arrive at a function $f_{N}$ which vanishes identically, and we thus obtain a decomposition

$$
f=\epsilon \sum_{k=1}^{N} \chi\left(S_{k}^{*}\right)
$$

We set

$$
g_{k}=N \epsilon \chi\left(S_{k}^{*}\right)=\chi\left(S_{k}^{*}\right),
$$

and we observe that, by (15) (and the corresponding formulas for $\left.S_{k}^{*}, k=2, \cdots, N\right)$

$$
\int_{X} g_{k} d \mu=\eta \mu(X)
$$

i.e., $g_{k} \in H$. Since, with $\alpha_{k}=\epsilon=N^{-1}$, (19) may be written in the form

$$
f=\sum_{k=1}^{N} \alpha_{k} g_{k}, \quad \sum_{k=1}^{N} \alpha_{k}=1,
$$

this shows that $f \in C(H)$, and Theorem II is proved.

4. As an illustration of the type of explicit inequality obtainable by means of Theorem I, we consider the eigenvalue problem (6) with the boundary conditions

$$
\begin{aligned}
u(a) & =u^{\prime}(a)=\cdots=u^{(n-1)}(a)=u^{(n)}(b) \\
& =u^{(n+1)}(b)=\cdots=u^{(2 n-1)}(b)=0 .
\end{aligned}
$$

If the coefficient $R(x)$ belongs to the class listed under (a) in $\S 3$, we have the following result.

THEOREM III. Let $\lambda=\lambda(R)=\lambda(R ; a, b)$ be the lowest eigenvalue of the differential equation 


$$
y^{(2 n)}-(-1)^{n} \lambda R(x) y=0
$$

with the boundary conditions (20), where $R>0, R \in L^{1}$ on $[a, b]$ and $n$ is a positive integer. If $R(x)$ is nonincreasing in $[a, b]$ then

$$
\lambda^{1 / p}(R) \int_{a}^{b}\left[(x-a)^{2 n} R(x)\right]^{1 / p} \frac{d x}{x-a} \geqq \frac{p}{2 n} \lambda^{1 / p}(1 ; 0,1)
$$

for any $p \geqq 1$. There will be equality in (22) whenever $R(x)$ coincides with a characteristic function $[a, t]$, where $t \in(a, b]$.

If we set

$$
d \sigma=(x-a)^{2(n / p)-1} d x,
$$

it follows from (8) that (22) will be established if we can show that

$$
\inf _{t \in(a, b]} \lambda^{1 / p}\left(\chi_{t}\right) \int_{a}^{b}\left[(x-a)^{2 n} \chi_{t}\right]^{1 / p} \frac{d x}{x-a}=\frac{p}{2 n} \lambda^{1 / p}(1 ; 0,1),
$$

where $\chi_{t}=\chi[a, t]$. Since

$$
\int_{a}^{b}\left[(x-a)^{2 n} \chi_{t}\right]^{1 / p} \frac{d x}{x-a}=\int_{a}^{t}(x-a)^{2(n / p)-1} d t=\frac{p}{2 n}(t-a)^{2 n / p},
$$

this will follow from the identity

$$
\lambda^{1 / p}\left(\chi_{t}\right)(t-a)^{2 n / p}=\lambda^{1 / p}(1 ; 0,1) .
$$

To establish (23) we note that, by an elementary argument,

$$
\lambda\left(\chi_{t} ; a, b\right)=\lambda(1 ; a, t) ;
$$

moreover, since $\lambda(1 ; a, t)=\inf \int_{\alpha}^{t}\left[u^{(n)}\right]^{2} d x\left[\int_{a}^{t} u^{2} d x\right]^{-1}$, where $u$ is subject to the boundary conditions (20) (with $b=t$ ), it is evident that $\lambda(1 ; a, t)=(t-a)^{-2 n} \lambda(1 ; 0,1)$. This completes the proof of Theorem III.

For $n=1$, we have $\lambda(1 ; 0,1)=\pi^{2} / 4$, and Theorem III yields the inequality

$$
\lambda^{1 / p}(R) \int_{a}^{b}\left[(x-a)^{2} R(x)\right]^{1 / p} \frac{d x}{x-a} \geqq \frac{p}{2}\left(\frac{\pi}{2}\right)^{2 / p} \quad(p \geqq 1)
$$

for the lowest eigenvalue of the problem

$$
y^{\prime \prime}+\lambda R(x) y=0, \quad y(a)=y^{\prime}(b)=0 .
$$

For $p=2$, this reduces to the known inequality [2]

$$
\lambda^{1 / 2}(R) \int_{a}^{b} R^{1 / 2}(x) d x \geqq \frac{\pi}{2} .
$$


5. If the coefficient $R(x)$ in (21) satisfies the condition $0 \leqq m \leqq R(x)$ $\leqq M<\infty$, an application of Theorem II leads to the following result.

THEOREM IV. Let $\lambda=\lambda(R)$ be the lowest eigenvalue of the differential equation (21) with the boundary conditions (20), where $0 \leqq m \leqq R(x)$ $\leqq M<\infty$ and $n$ is a positive integer. If the number $\eta$ is defined by

$$
\int_{a}^{b} R^{1 / p}(x) d x=(b-a)\left[M^{1 / p} \eta+m^{1 / p}(1-\eta)\right], \quad(0 \leqq \eta \leqq 1),
$$

then

$$
\lambda(R) \geqq \lambda\left(R_{0}\right),
$$

where $R_{0}=m$ for $a \leqq x<a \eta+b(1-\eta)$ and $R_{0}=M$ for $a \eta+b(1-\eta)$ $\leqq x \leqq b$.

By (8) and Theorem II,

$$
\lambda^{1 / p}(R) \int_{a}^{b} R^{1 / p} d x \geqq \inf \lambda^{1 / p}(T) \int_{a}^{b} T^{1 / p} d x,
$$

if $T$ ranges over the class of functions $T=m+(M-m) \chi\left(X_{0}\right)$, and $X_{0}$ is a subset of $[a, b]$ of Lebesgue measure $\eta(b-a)$, where $\eta$ is defined in (24). Since

$$
\int_{a}^{b} R^{1 / p} d x=\int_{a}^{b} T^{1 / p} d x
$$

we thus have

$$
\lambda(R) \geqq \inf \lambda(T) .
$$

If $y_{R}$ is the solution of (21)-(20) associated with the lowest eigenvalue, it is well known that $y_{R}^{2}$ is nondecreasing in $[a, b]$ if $R$ is nonnegative. Since, for a nondecreasing $y^{2}$, the value of

$$
\int_{a}^{b}\left[m+(M-m) \chi\left(X_{0}\right)\right] y^{2} d x
$$

is largest if $X_{0}$ is the interval $[a \eta+b(1-\eta), b]$, it follows that

$$
\frac{1}{\lambda(T)}=\int_{a}^{b} T y_{T}^{2} d x \leqq \int_{a}^{b} R_{0} y_{T}^{2} d x \leqq \frac{1}{\lambda\left(R_{0}\right)} .
$$

In view of (25), this proves Theorem IV.

For $n=1, p=1$, Theorem IV reduces to a result of Krein [1]. 


\title{
REFERENCES
}

1. M. G. Krein, On certain problems on the maximum and minimum of characteristic values and on the Lyapunov zones of stability, Prikl. Mat. Meh. 15 (1951), 323-348.

2. Z. Nehari, Some eigenvalue estimates, J. Analyse Math. 7 (1959), 79-88.

Carnegie Institute of Technology

\section{WEAK CONVERGENCE OF THE SEQUENCE OF SUCCESSIVE APPROXIMATIONS FOR NONEXPANSIVE MAPPINGS}

BY ZDZISEAW OPIAL

\author{
Communicated by F. Browder, September 26, 1966
}

In a recent paper [4] F. E. Browder and W. V. Petryshyn have shown that if a nonexpansive mapping $T: X \rightarrow X$ of a Hilbert space $X$ into itself is asymptotically regular and has at least one fixed point then, for any $x$ in $X$, a weak limit of a weakly convergent subsequence of the sequence of successive approximations $\left\{T^{n} x\right\}$ is a fixed point of $T$. The main object of the present note is to strengthen considerably this result by showing that under the same assumptions the sequence $\left\{T^{n} x\right\}$ is necessarily weakly convergent.

In $\$ 1$ we recall some basic definitions and prove two simple lemmas. In $\$ 2$ we prove the weak convergence of the sequence $\left\{T^{n} x\right\}$ and in $\$ 3$ we discuss the possibility of the extension of this result to Banach spaces having weakly continuous duality mappings. In $\$ 4$ an application of Theorem 2 stated in $\$ 3$ to a modified sequence of successive approximations is given and, in $\$ 5$, limits of validity of the first key lemma of $\$ 1$ are discussed.

1. Let $C$ be a convex closed set in a Banach space $X$. A mapping $T: C \rightarrow X$ is called nonexpansive if $\|T x-T y\| \leqq\|x-y\|$ for any $x, y$ in $C$. Following [4], a mapping $T: C \rightarrow C$ is said to be asymptotically regular if, for any $x$ in $C$, the sequence $\left\{T^{n+1} x-T^{n} x\right\}=\left\{(I-T)\left(T^{n} x\right)\right\}$ tends to zero as $n \rightarrow \infty$. Finally, a mapping $T: C \rightarrow X$ is called demiclosed if its graph in $C \times X$ is closed in the topology of a Cartesian product induced in $C \times X$ by the weak topology in $C$ and the strong topology in $X$; i.e., if for any sequence $\left\{x_{n}\right\} \subset C$ which converges weakly to an $x_{0}$ in $C$, the strong convergence of the sequence $\left\{T x_{n}\right\}$ to a $y_{0}$ in $X$ implies that $T x_{0}=y_{0}$. 